\title{
Estrutura, metabolismo e funções fisiológicas da lipoproteína de alta densidade
}

\section{Structure, metabolism and physiologic functions of high-density lipoproteins}

Emerson Silva Lima'; Ricardo David Couto ${ }^{2}$

Unitermos
HDL
Doença arterial coronariana
Transporte reverso do colesterol

\section{resumo}

Diversos estudos clínicos, epidemiológicos e experimentais têm mostrado de maneira incontestável a relação entre dosagem sérica dos níveis de lipoproteína de alta densidade (HDL) e doença cardiovascular. Baixos níveis de HDL estão presentes em aproximadamente $10 \%$ da população e representam um dos mais freqüentes achados de dislipidemia nos pacientes com doença arterial coronariana (DAC). Esses níveis reduzidos de HDL poderiam ser incapazes de efetivamente eliminar o excesso de colesterol das paredes vasculares, contribuindo para o fenômeno inflamatório que caracteriza a patogênese da aterosclerose nas suas fases iniciais. Outros inúmeros estudos têm convincentemente mostrado que a HDL também exerce efeitos diretos sobre os processos inflamatórios, por exemplo, através da modulação da expressão de diversas proteínas de fase aguda. Além disso, a HDL também possui diversos outros efeitos antiaterogênicos, como efeitos antioxidantes, inibição da agregação plaquetária e da migração de monócitos. O presente artigo faz uma revisão da literatura atual sobre o metabolismo da HDL e suas principais ações na prevenção da doença arterial coronariana.

Several experimental, clinical and epidemiological researches have shown the incontestable causal relationship between low high-density lipoprotein (HDL) plasma concentrations and cardiovascular pathology on an atherosclerotic basis. Low HDL levels characterize about $10 \%$ of the general population and they represent the most frequent dyslipidemia in patients with coronary artery disease. Reduced HDL concentrations would be unable to effectively eliminate the cholesterol excess at the vascular wall, contributing to the inflammatory phenomenon that characterizes the pathogenesis of atherosclerosis since its initial phases. Results of numerous studies reasonably allow supposing that HDL is able to exert, also directly, anti-inflammatory actions through the modulation of expression of diverse acute phase proteins. Furthermore, HDL also exerts several other atheroprotective effects, such as antioxidants affects, inhibition of platelets aggregation and monocytes migration. This paper is a review on recent literature data about HDL metabolism and its role in the prevention of coronary artery disease. 


\section{Introdução}

Numerosos estudos clínicos e epidemiológicos têm relacionado fortemente a diminuição da concentração plasmática da lipoproteína de alta densidade (HDL) com o desenvolvimento de doença arterial coronariana (DAC) ${ }^{(96)}$. Baixa concentração de $\mathrm{HDL}$ circulante é um fator de risco independente para $\mathrm{DAC}^{(3)}$. Esse efeito também é observado na síndrome metabólica ${ }^{(1,8,9,72)}$, que inclui resistência à insuli$n a^{(68)}$, intolerância à glicose ${ }^{(7,47,94)}$ e hipertensão arterial ${ }^{(25,75)}$. Além disso, o aumento das concentrações plasmáticas das lipoproteínas aterogênicas, incluindo a lipoproteína de muito baixa densidade (VLDL) e a lipoproteína de baixa densidade (LDL), está freqüentemente associado à diminuição da concentração da HDL. O efeito antiaterogênico da HDL se dá, sobretudo, devido à sua propriedade de transportar lípides, principalmente ésteres de colesterol, dos tecidos periféricos para o fígado, o que é conhecido como transporte reverso do colesterol (TRC). Contudo, outras ações protetoras importantes da HDL, além do TRC, têm sido descritas em diversos modelos experimentais e estudos epidemiológicos. Essas ações incluem: proteção antioxidante, mediação do efluxo de colesterol, inibição da expressão de moléculas de adesão celular, ativação de leucócitos, indução da produção de óxido nítrico (NO), regulação da coagulação sangüínea e da atividade plaquetária.

Este trabalho faz uma revisão das principais características antiaterogênicas da HDL, além do transporte reverso do colesterol, e suas implicações na prevenção de doenças ateroscleróticas.

\section{Definição e estrutura}

A fração lipoprotéica conhecida como HDL é, na verdade, constituída por um grupo de partículas originalmente obtidas por ultracentrifugação do plasma, com densidade entre 1,063 e 1,25g/ml. Assim, as HDL são as menores (7$17 \mathrm{~nm}$ de diâmetro) e as mais densas $(1,063<\mathrm{d}<1,25 \mathrm{~g} / \mathrm{ml})$ das frações lipoprotéicas plasmáticas ${ }^{(32,39,77)}$. Sua estrutura consiste em um núcleo lipídico hidrofóbico, contendo principalmente ésteres de colesterol, uma pequena quantidade de triacilgliceróis e colesterol não-esterificado. Esse núcleo é circundado por uma camada monofásica de fosfolipídios, colesterol não-esterificado e apolipoproteínas. As principais apolipoproteínas da HDL são apoA-I e apoA-II, embora outras, como apoA-IV, apoA-V, apoC-I, apoC-II, apoC-III, apoD e apoE, possam estar presentes ${ }^{(39)}$. A HDL também serve como transportador plasmático eficaz de proteínas envolvidas com o metabolismo lipídico, como proteína transportadora de éster de colesterol (CETP), lecitina, colesterol aciltransferase (LCAT) e proteína transportadora de fosfolipídios (PLTP), que participam ativamente das vias metabólicas da $\mathrm{HDL}{ }^{\left({ }^{89}\right)}$. Essas e outras moléculas de origem protéica presentes na HDL estão descritas na Tabela 1.

Quando isoladas por ultracentrifugação, podem ser observadas duas frações predominantes de HDL: uma subfração principal, chamada $\mathrm{HDL}_{2}$ encontrada na faixa de densidade entre 1,063 e 1,125g/ml, e outra, chamada $\mathrm{HDL}_{3}$, obtida entre 1,125 e 1,21 $/ \mathrm{ml}$. Uma terceira fração obtida em menor quantidade, conhecida como lipoproteína de densidade muito alta (VHDL), pode também ser isolada na faixa de densidade entre 1,21 e 1,25(35). Quando separada por eletroforese em sistema de gel de poliacrilamida não-desnaturante, que usa o tamanho e a carga da partícula como base para a separação, a fração da HDL previamente obtida por ultracentrifugação pode ser dividida em cinco subfrações distintas ${ }^{(65,66,92)}$. Essas subfrações separadas por ordem decrescente de tamanho são: $\mathrm{HDL}_{2 \mathrm{~b}}$ (diâmetro médio $10,6 \mathrm{~nm}), \mathrm{HDL}_{2 \mathrm{a}}(9,2 \mathrm{~nm}), \mathrm{HDL}_{3 \mathrm{a}}(8,4 \mathrm{~nm}), \mathrm{HDL}_{3 \mathrm{~b}}(8 \mathrm{~nm}) \mathrm{e}$ $\mathrm{HDL}_{3 \mathrm{c}}(7,6 \mathrm{~nm})$, que também podem ser identificadas por ressonância magnética nuclear (RMN), técnica que tem sido mais recentemente utilizada na identificação de subfrações da HDL em função do tamanho das partículas ${ }^{(59)}$.

As HDL também podem ser divididas em duas subpopulações, tendo como base a sua composição de apolipoproteínas: uma subpopulação compreendendo as partículas contendo somente apoA-I (HDL A-I) e outra que contém as duas apolipoproteínas (HDL A-I/A-II). Enquanto a proporção de apoA-l é de aproximadamente $50 \%$ nas HDL A-I e HDL A-I/A-II, na maioria dos indivíduos quase toda a apoA-ll está na fração das HDL A-I/A-II. A maior parte das HDL A-I/A-II está na fração de densidade $\mathrm{HDL}_{3^{\prime}}$ e as $\mathrm{HDL} A$-I são os componentes principais das frações $\mathrm{HDL}_{3}$ e $\mathrm{HDL}_{2}{ }^{(77,89)}$.

Quando as partículas de HDL são separadas por eletroforese em gel de agarose, ocorrem quatro bandas de migração denominadas alfa, pré-alfa, pré-beta e gama de acordo com as bandas observadas na separação do plasma. As partículas que migram na posição alfa são esféricas e constituem a maior proporção, na qual estão incluídas as frações $\mathrm{HDL}_{3}$ e $\mathrm{HDL}_{2}$, assim como $\mathrm{HDL}$ A-I e $\mathrm{HDL}$ A-I/A-II. As partículas conhecidas como pré- $\beta_{1} \mathrm{HDL}$ são, na verdade, moléculas de apoA-I ou partículas discóides consistindo de uma ou duas moléculas de apoA-I contendo fosfolipídios e, possivelmente, uma pequena quantidade de colesterol não-esterificado. A fração gama é constituída de partículas que contêm apoE e não apoA-( ${ }^{(4,92)}$. 


\begin{tabular}{|c|c|c|}
\hline Proteina/enzima & Sigla & Funções \\
\hline Apolipoproteína A-I & ApoA-I & $\begin{array}{l}\text { Lipoproteína estrutural da HDL representa o maior componente } \\
\text { protéico dessa partícula. É responsável por ativação da LCAT, } \\
\text { estimulação do efluxo do colesterol e ligação aos receptores da } \\
\text { HDL (SR-BI, ABCA-1). Pode ser também responsável pela ação } \\
\text { antioxidante da HDL }\end{array}$ \\
\hline Apolipoproteína A-II & ApoA-II & Modula a atividade de LCAT, CETP e LH. Possui ação antioxidante \\
\hline Apolipoproteína A-IV & ApoA-IV & $\begin{array}{l}\text { Ativação da LCAT, modulação da lipase lipoprotéica e estimulação } \\
\text { do efluxo de colesterol }\end{array}$ \\
\hline Apolipoproteína E & ApoE & Ligante dos receptores de ApoE \\
\hline Apolipoproteína F & ApoF & Inibição da CETP \\
\hline Apolipoproteína J & ApoJ & Clusterina participa da função da PON-1 \\
\hline Lecitina colesterol aciltransferase & LCAT & Esterificação do colesterol livre e maturação do HDL \\
\hline $\begin{array}{l}\text { Proteína de transferência } \\
\text { de ésteres de colesterol }\end{array}$ & CETP & $\begin{array}{l}\text { Modula a troca de ésteres de colesterol e triglicérides entre HDL e } \\
\text { lipoproteínas contendo ApoB. Geração de ApoA-I pobre em lípides }\end{array}$ \\
\hline $\begin{array}{l}\text { Proteína de transferência } \\
\text { de fosfolípides }\end{array}$ & PLTP & $\begin{array}{l}\text { Transferência de fosfolípides de lipoproteínas ricas em triglicerídeos } \\
\text { para HDL e geração de ApoA-I pobre em lípides }\end{array}$ \\
\hline Paraoxonase & PON-1 & $\begin{array}{l}\text { Hidrolisa hidroperóxidos de fosfolípides na superfície da partícula } \\
\text { de LDL, inibindo assim a oxidação dessa partícula in vivo }\end{array}$ \\
\hline
\end{tabular}

\section{Síntese e metabolismo}

As HDL são sujeitas a um processo de remodelamento contínuo no compartimento plasmático. As apoA-l e apoA-II são sintetizadas principalmente no fígado, apesar de uma proporção da apoA-I ser também formada no

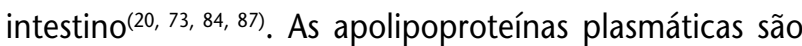
lipidadas por processo dependente de proteína específica conhecida como ATP binding cassette transporter A1 (ABCA-1) ${ }^{(19,57,76,80,82,90,91)}$, formando uma partícula de HDL discóide contendo, além da apolipoproteína, fosfolipídios e uma pequena quantidade de colesterol não-esterificado. Essas partículas discóides adquirem colesterol não-esterificado de outras lipoproteínas e das membranas celulares por um processo passivo independente de ABCA-1 e também são excelentes substratos para a ação da LCAT, que esterifica o colesterol. Esse processo tende a formar partículas esféricas de núcleo lipídico, contendo principalmente ésteres de colesterol (Figura 1). O maior número de partículas de HDL esféricas encontradas no plasma pode ser explicado em função da relação entre a eficiência de esterificação da LCAT e o tempo de permanência da HDL na circulação ${ }^{(4)}$.

A remoção das HDL do plasma se dá por processo complexo. A maioria dos constituintes é removida separadamente e apenas uma fração da HDL é metabolizada como

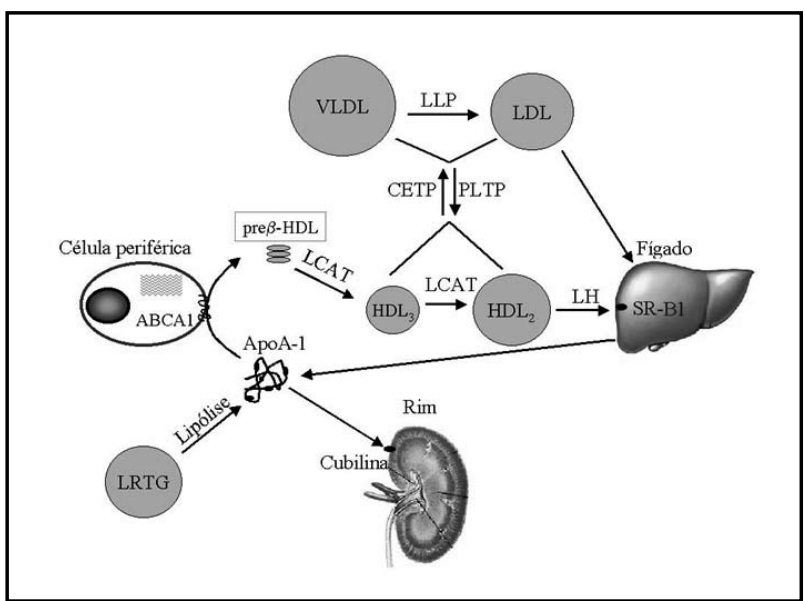

Figura 1 - Principais vias do transporte reverso do colesterol e metabolismo da HDL

partícula intacta ${ }^{(2)}$. Por exemplo, os ésteres de colesterol são transferidos para as VLDL e LDL, por ação da CETP, ou são seletivamente captados pelo fígado, em um processo dependente de receptores ligantes de HDL, sendo o principal deles o SR-B1 ${ }^{(87)}$. Os triacilgliceróis e fosfolipídios são removidos por hidrólises catalisadas por lipases, entre as quais lipase hepática (LH), lipase endotelial e fosfolipase $A_{2}$. A apoA-I da HDL é metabolizada independentemente, após sua dissociação da partícula durante o processo metabólico. A apoA-I dissociada pode ser tanto reutilizada na formação de uma nova partícula 
de HDL quanto excretada através dos rins. As HDL contendo somente apoA-I são catabolisadas de forma mais rápida do que aquelas contendo apoA-I e apoA-II(20).

O processo metabólico da HDL tem um papel importante na composição, na forma, no tamanho e na carga de superfície das partículas, sendo o responsável pela heterogeneidade das HDL plasmáticas. O fenômeno de remodelamento plasmático ocorre mais rapidamente do que o tempo de permanência da HDL na circulação (três a cinco dias), sendo o principal responsável pelo metabolismo dessa fração lipoprotéica ${ }^{(8)}$. Um dos resultados do remodelamento das HDL por fatores como CETP, LH e PLTP é a formação de apoA-1 pobre em lípides que pode ser reincorporada na formação de novas partículas de HDL ou excretada na urina. Uma vez formada, a apoA-I pobre em lípides captará o colesterol celular através do ABCA-1, dando início à formação de uma nova partícula de $\mathrm{HDL}^{(20)}$. Assim, a geração de apoA-I pobre em lípides é um fator determinante da taxa de efluxo do colesterol celular (Figura 1).

\section{Catabolismo das apolipoproteínas}

O mecanismo de remoção da circulação das proteínas presente na HDL é bem menos compreendido do que aquele que ocorre com os lipídios associados à partícula. $\mathrm{O}$ principal mecanismo de retirada dessas apolipoproteínas é a captação via receptores presentes no fígado e rins, assim como na placenta e no saco amniótico durante a gravidez. Pequenas partículas de HDL de tamanho menor que $8 \mathrm{~nm}$ e apoA-I livre de lipídios são filtradas pelos glomérulos renais e posteriormente reabsorvidas no túbulo contorcido proximal $^{(2,89)}$.

A cubilina, receptor do fator intrínseco/vitamina $B_{12}$, foi recentemente identificada com receptora de apoA-I da HDL em células epiteliais do túbulo contorcido proximal renal e do saco amniótico ${ }^{(5,21,50)}$. Em indivíduos nos quais essa proteína encontra-se deficiente, há aumento da excreção de apoA-I na urina. Apesar disso, a cubilina não possui domínio transmembrânico e não poderia mediar a internalização de ligantes sem o auxílio de co-receptores. Portanto, a megalina, um membro da família do gene do receptor da LDL, tem sido indicada como responsável por esse papel nos rins e na placenta ${ }^{(22)}$. Após internalização, os ligantes da cubilina (HDL, apoA-I e complexo fator intrínseco/vitamina $B_{12}$ ) são degradados por lisossomos endógenos. Portanto, é improvável que a apoA-I captada pelas células renais retorne ao compartimento plasmático e que somente a cubilina seja fator determinante na concentração da HDL plasmática.
As HDL que contêm apoE constituem a minoria das partículas de HDL e são internalizadas pelo fígado via receptores de LDL. Também existem evidências da presença de receptores hepáticos de HDL não contendo apoE. Alguns estudos têm identificado sítios de ligação para HDL de diferentes tamanhos em células hepáticas, os quais são candidatos a mediadores da captação da partícula de HDL como um todo(4). Estudos morfológicos também têm fornecido evidência da ligação e endocitose da HDL no fígado. Também alguns autores têm demonstrado ressecreção de HDL que tinha sido internalizada por células hepáticas ${ }^{(20)}$. Interessantemente, ligação hepática, captação, degradação e ressecreção de HDL estão alteradas em camundongos homozigóticos deficientes em leptina ${ }^{(38)}$. Esses animais possuem níveis plasmáticos elevados de $\mathrm{HDL}$, devido ao catabolismo mais lento dessa partícula. Tall et al. ${ }^{(85)}$ sugerem a presença de um receptor hepático para HDL regulado pela leptina, o qual regula os níveis de HDL mediando a captação da partícula pelas células hepáticas.

\section{Funções fisiológicas}

\section{Transporte reverso do colesterol}

O transporte reverso do colesterol é a via pela qual o colesterol nos tecidos periféricos é transferido através do plasma para o fígado. Este também pode ser reciclado ou excretado na bile e/ou utilizado como arcabouço para produção de hormônios, respectivamente. Para isso são necessários: 1) a transferência de fosfolipídios e colesterol de membranas celulares para partículas lipoprotéicas ricas em proteínas e pobres em lipídios, através do espaço extracelular por processo dependente de ABCA-1 que resulta na formação

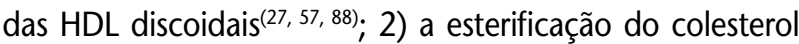
mediada pela LCAT da HDL discoidal, transformando-a em partícula esférica; 3) a interação das HDL esféricas com a proteína transportadora de colesterol éster (CETP), que transfere o conteúdo de ésteres de colesterol (EC), ou parte dele, para VLDL e seus remanescentes; e 4) a recirculação do EC para o fígado, tanto pela remoção de $\beta$-VLDL por meio dos receptores da lipoproteína de baixa densidade quanto pela remoção de partículas de HDL pelos receptores da família SR-BI, que removem seletivamente os EC das partículas de HDL e também de LDL para os hepatócitos e células produtoras de hormônios esteroidais, sem internalizar as proteínas da HDL, os fosfolipídios e as apolipoproteínas ${ }^{(20)}$.

Embora os mecanismos nos quais ocorre dissociação de lipídios e lipoproteínas e incorporação dos EC nas membra- 
nas plasmáticas não estejam ainda totalmente elucidados, acredita-se que exista a formação de um canal hidrofóbico por onde o colesterol da HDL se difunde para a membrana plasmática. O processo realizado pelos receptores da família do receptor de LDL (LDL-r) ou scavengers classe A é diferente, ou seja, acontece a internalização das lipoproteínas via endossomos, seguida da fusão com lisossomos para degradação das partículas lipoprotéicas. É importante enfatizar que o TRC é realizado por um conjunto de partículas efetoras como apoproteínas, lipídios, proteínas de transferência, enzimas, entre outras, associadas ou não a HDL e suas subclasses, sendo esse um grande desafio para a lipidologia: entender como a relação influxo/efluxo do colesterol pode ser modulada para prevenir a aterosclerose ${ }^{(88)}$.

\section{Ação antioxidante}

A oxidação da LDL é considerada o principal evento de iniciação do desenvolvimento da aterosclerose $\mathrm{e}^{(13,28,37,83,95)}$. A LDL oxidada (LDL-ox) age como fator quimiotático para monócitos que, transformados em macrófagos túrgidos com lipídios (células espumosas), exercem efeitos citotóxicos sobre as células endoteliais, aumentando a ativação de plaquetas, estimulando a migração e a proliferação de células musculares lisas (SMC) e antagonizando os efeitos vasodilatadores do óxido nítrico ${ }^{(13)}$. Diversos autores têm mostrado que a HDL reduz significativamente as modificações oxidativas da $\operatorname{LDL}^{(20,31,42,45,46)}$.

A HDL pode inibir a oxidação da LDL quando causada por íons de metais de transição e prevenir a formação de peróxidos lipídicos pela 12-lipoxigenase ${ }^{(20,26,31,42,45,46,74,79)}$. A Figura 2 mostra o resultado de um experimento clássico ilustrando esse efeito. Nele a HDL foi capaz de inibir a oxidação da LDL induzida por sulfato de cobre $\left(\mathrm{CuSO}_{4}\right)$. A HDL também adquire alguns produtos de oxidação da LDL como, por exemplo, lisofosfatidilcolina e peróxidos lipídicos, transportando-os até o fígado, onde serão metabolizados $^{(26)}$. A inibição da oxidação da LDL pela HDL é comumente atribuída ao seu conteúdo de antioxidante ( $\alpha$-tocoferol, licopeno, estrógenos) $)^{(6,61,62)}$, às propriedades antioxidantes da apoA-I e apoA-II e, principalmente, devido à presença de paraoxonase, uma enzima que catalisa a hidrólise de ácidos carboxílicos aromáticos e compostos organofosforados ${ }^{(10,12,44,63,78,93)}$.

A paraoxonase também catalisa a quebra de fosfolipídios oxidados na $L D L$, os quais estimulam a produção de citocinas e induzem a adesão de monócitos na superfície de células endoteliais ${ }^{(93)}$. Além disso, diminui o conteúdo de peróxidos lipídicos em artérias coronárias humanas e lesões

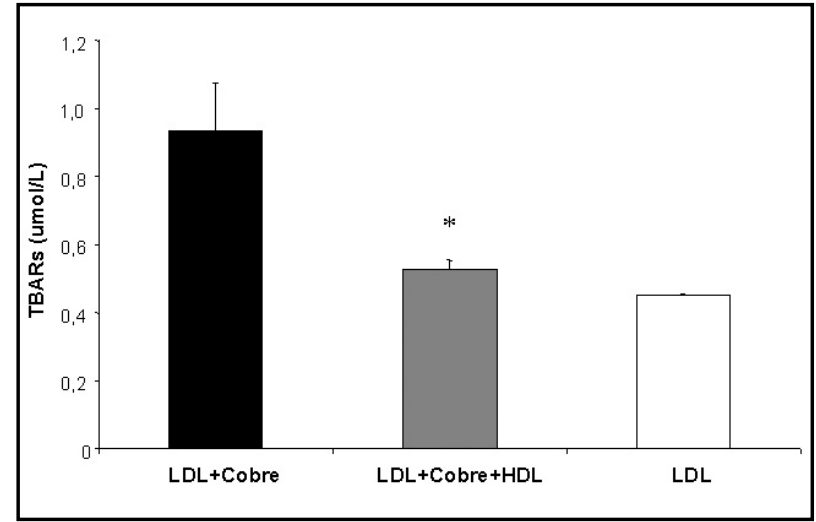

Figura 2 - Ação antioxidante in vitro da HDL. Em tubos de ensaio uma solução de LDL $\left(0,25 \mathrm{mg}\right.$ de proteína/ml) foi incubada isoladamente, após adição de $1 \mathrm{mM} \mathrm{de} \mathrm{CuSO}_{4}$ ou com uma solução de $\mathrm{HDL}$ (1 $\mathrm{mg}$ de proteína $/ \mathrm{ml}$ ) por uma hora a $37^{\circ} \mathrm{C}$. Em seguida foi realizado teste das substâncias reativas ao ácido tiobarbitúrico (TBARs) com o objetivo de mensurar produtos da oxidação de lipídios presentes nas lipoproteínas. *Diferente da LDL incubada somente com $\mathrm{CuSO}_{4^{\prime}}(p<0,05$, teste $t)$

da carótida ${ }^{(18)}$. Em animais susceptíveis à aterosclerose, como camundongos deficientes em apoE ou receptores de LDL, altos níveis de marcadores de oxidação estão acompanhados da diminuição da atividade da paraoxonase ${ }^{(78)}$. Tal diminuição também foi observada em animais submetidos a uma dieta aterogênica e em pacientes diabéticos com níveis elevados de hemoglobina glicada ${ }^{(71)}$. Por outro lado, a expressão da apoA-I humana em camundongos aumentou a atividade da paraoxonase ${ }^{(78)}$. Além disso, diversos estudos genéticos têm confirmado a importância da paraoxonase na inibição do desenvolvimento da aterosclerose. Existem três genes identificados que codificam enzimas com atividade paraoxonase: PON1, PON2 e PON3. O polimorfismo localizado na posição 192 da PON1 é um importante determinante da atividade dessa enzima ${ }^{(41)}$. A isoforma-A que possui glutamina nessa posição tem atividade oito vezes mais baixa que a isoforma- $B$ que possui arginina. Além disso, diversos estudos têm mostrado o aumento do risco de DAC em indivíduos com baixa atividade de paraoxonase ${ }^{(43)}$.

\section{Estímulo da produção de óxido nítrico}

O endotélio vascular tem papel crucial no aparecimento e no desenvolvimento da aterosclerose. Fatores importantes da disfunção endotelial são a diminuição da biodisponibilidade do NO e o aumento da afinidade do endotélio a leucócitos que estão associados aos eventos iniciais do processo aterogênico ${ }^{(2,11,23)}$. Diversos estudos in vivo têm mostrado efeitos benéficos da HDL na função endotelial ${ }^{(34,40,48,56,67,97)}$. Por exemplo, a correlação negativa entre vasodilatação dependente de $\mathrm{NO}$ e níveis plasmáticos de HDL tem sido descrita, assim como a melhora da função endotelial na hipercolesterolemia humana após infusão de 


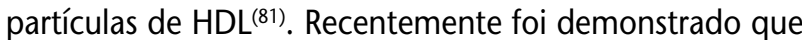
HDL é um fator autônomo de proteção para o endotélio, induzindo a ativação da NO sintase, a liberação de NO e o efeito de vasorrelaxamento ${ }^{(67)}$.

Os efeitos protetores da HDL à função endotelial estão associados a sua ligação com os receptores SR-B1 e podem estar relacionados a substâncias reguladoras presentes na composição da partícula de HDL. Esses efeitos seriam mediados por uma proteinoquinase, a qual pode ser ativada após a ligação da HDL com os receptores SR-B1 e a conseqüente interação de lisoesfingolipídios presentes na HDL com genes receptores de diferenciação presentes na membrana da célula endotelia|(49,67).

\section{Inibição da expressão de moléculas de adesão e ativação de leucócitos}

As partículas de $\mathrm{HDL}$ interferem em várias das inúmeras funções secretoras das células endoteliais. A prostaglandina $\left(\mathrm{PGI}_{2}\right)$, produzida nas células endoteliais por ação das ciclooxigenases (COX-2), tem alta atividade vasorrelaxante, inibe a ativação plaquetária e diminui a liberação de fatores de crescimento que agem estimulando a proliferação local das células musculares lisas ${ }^{(14)}$. A HDL, em concentrações fisiológicas, estimula a produção de $\mathrm{PGl}_{2}$. O efeito estimulante depende de dois fatores: o suprimento de ácido araquidônico para as células endoteliais, a partir das partículas de HDL, e a indução da síntese de COX-2, que é a principal via de produção de prostanóides pelas células endoteliais ${ }^{(64)}$.

A HDL também demonstra ter importante papel na modulação da síntese do peptídeo $C$ natriurético (CNP), o qual causa vasodilatação, inibe a proliferação das SMC e da secreção de endotelina-1. A placa aterosclerótica sofre forte influência dos tipos celulares nela contidos. Tem sido relatado que a adesão de leucócitos às células endoteliais e sua interação com as SMC possuem papel crucial no desenvolvimento da placa aterosclerótica. A adesão celular é mediada por moléculas de adesão presentes na superfície das células do endotélio vascular, representadas, basicamente, pelas moléculas de adesão vascular VCAM-1, ICAM-1 e pelas selectinas $P$, L e E. O processo de adesão dos leucócitos ao endotélio é chamado de marginação, seguido pela fase de ativação da ligação ao endotélio e finalmente à fase de migração, em que os leucócitos tornam-se aptos a migrar através dos tecidos. Essas moléculas de adesão (necessárias para a aderência dos leucócitos às células endoteliais), principalmente VCAM-1, ICAM-1 e selectina-E, estão abundantemente presentes nas placas ateroscleróticas ${ }^{(33)}$. Cybulsky et al. ${ }^{(16)}$ observaram pela pri- meira vez o aumento da expressão de VCAM-1 em animais hipercolesterolêmicos, tanto devido à indução por dietas hipercolesterolêmicas quanto pela deficiência do LDL-r. A expressão de moléculas de adesão é induzida por citocinas (IL-1 e TNF- $\alpha$ ), que são liberadas por células ativadas, por lisofosfatidilcolina presente em LDL oxidada e por produtos de lipólise. A modulação da HDL sobre as moléculas de adesão, especialmente a VCAM-1 pode ser dependente da sua composição fosfolipídica, aspecto verificado em partículas reconstituídas após depleção de fosfolipídios ${ }^{(16)}$. Mesmo após a remoção das partículas de HDL das culturas celulares, o efeito inibitório permanece, excluindo então o efeito per se de moléculas antioxidantes presentes nessas partículas. Esse fenômeno parece estar relacionado à indução de fatores de transcrição nuclear (ex.: NF-אB), visto que a HDL não inibe translocação nuclear, ligação com seqüências específicas do DNA e degradação ou síntese do NF-kB.

Apesar de todas essas evidências, o papel da HDL na indução da supressão de moléculas de adesão para prevenir a aterosclerose é ainda uma questão importante para debate.

\section{Regulação do processo de coagulação e fibrinólise}

Estudos epidemiológicos têm demonstrado a associação de coagulação e fibrinólise com doença arterial coronariana $(D A C)^{(86)}$. O estudo sobre doença cardíaca de NorthwickPark demonstrou que a ação pró-coagulante do fator VII, fator dependente de vitamina K, é um potente preditor da mortalidade por DAC ${ }^{(58)}$. Altos níveis do inibidor tipo I do ativador do plasminogênio (PAI-I) também estão associados ao aumento do risco de eventos cardiovasculares ${ }^{(36)}$. Tais achados sugerem que o desequilíbrio entre a coagulação e a fibrinólise pode conduzir à aterosclerose.

A ativação dos fatores da coagulação é seguida pela produção da tenase extrínseca (complexo formado por fator tecidual, fator VIla, fosfolipídios e íons cálcio) e protrombinase (complexo de fatores da coagulação como Va, Xa, II, fosfolipídeos e íons cálcio). Essas ativações são moduladas pelas partículas de HDL. Diferente das lipoproteínas aterogênicas, como LDL e VLDL, as quais estimulam a secreção de TF e a ativação da tenase extrínseca, a HDL per se não estimula a secreção de TF a partir de células endoteliais e monócitos, sendo a síntese de TF estimulada pela VLDL e inibida pela $\mathrm{HDL}^{(55)}$. Estudos recentes demonstraram que a atividade inibitória da HDL sobre a ativação desses fatores deve-se à presença do fator de inibição tecidual (TPFI) presente nessa lipoproteína ${ }^{(17)}$. Moyer et al. ${ }^{(51)}$ demonstraram que, embora 
a trombina seja gerada na superfície das partículas da $\mathrm{HDL}$, esse processo é 20 vezes menos expressivo do que quando ela é gerada na superfície das partículas de lipoproteínas ricas em triglicérides (TG). Verificou-se também aumento da atividade da protrombinase em dislipidemias onde há maior concentração de lipoproteínas ricas em TG e diminuição da HDL plasmática ${ }^{(51)}$.

A partícula de HDL também tem efeito anticoagulante através do estímulo da proteína $C$ ativada (APC), que tem importante papel na inativação proteolítica dos fatores Va e VIlla da coagulação. O aumento da atividade da APC é ainda maior devido ao estímulo da proteína $S$ pela $\mathrm{HDL}$, ou seja, há um efeito anticoagulante sinérgico entre as proteínas C e S. Essa característica anticoagulante da HDL é devida à presença de anticoagulantes naturais, como cardiolipina e fosfatidiletanolamina. A HDL parece, a priori, participar também do processo de fibrinólise, pois, embora a secreção do PAI-1 esteja aumentada (aumento da secreção pelas células endoteliais) tanto na hipercolesterolemia quanto na hipertrigliceridemia, a concentração da HDL plasmática é inversamente proporcional às concentrações de PAI-1 e do ativador tecidual de plasminogênio (Tpa). Essa associação pode refletir o efeito in vitro inibitório da HDL na secreção dos fatores PAI-1 e tPA pelo endotélio(15). A modulação do processo de coagulação e de fibrinólise pela HDL é acompanhada da inibição da secreção de citocinas, TNF- $\alpha$ e IL-1 que aumentam tanto a coagulação quanto a fibrinólise.

\section{Inibição da ativação plaquetária}

A HDL baixa é preditor independente da formação aguda de trombo dependente da ativação plaquetária( ${ }^{(15)}$. Estudos in vitro demonstraram que, na presença ou na ausência de plasma rico em plaquetas ou apenas em plaquetas isoladas, a HDL inibe a ligação do fibrinogênio induzida por trombina na superfície plaquetária. A HDL inibe ainda a trombina e a formação de ADP devido ao estímulo à secreção de grânulos alfa e grânulos densos nas plaquetas ${ }^{(53)}$.

Em estudos utilizando inibidores da NO sintase, obteve-se diminuição da capacidade inibitória da agregação plaquetária pela HDL. Por outro lado, a presença de precursores de NO aumenta a capacidade inibitória da HDL sob a ativação plaquetária. A inibição da agregação plaquetária é devida, provavelmente, à indução da síntese de óxido nítrico (NO) pela apoE presente nessas lipoproteínas ${ }^{(70)}$. Por outro lado, a maior parte dos estudos que avaliaram o fenômeno inibitório da ativação plaquetária utilizou partículas de HDL derivadas de pacientes deficientes em apo $E^{(24,69)}$. Verificou-se também que, após incubação da HDL com plaquetas, há presença de um substrato (proteína fosforilada com peso molecular de $43 \mathrm{kDa}$ ) para a proteinoquinase $\mathrm{C}^{(52)}$.

A ativação da proteinoquinase $C$ pela $\mathrm{HDL}$ induz $\mathrm{o}$ antiporte sódio-próton $\left(\mathrm{H}^{+}\right)$que gera a alcalinização do compartimento citoplasmático, impedindo a liberação de cálcio $\left(\mathrm{Ca}^{2+}\right)$ a partir de organelas intracelulares armazenadoras. Esse desfecho, por sua vez, conduz à inibição da ativação plaquetária. Ainda, a ativação da proteinoquinase $C$ inibe a fosfolipase $C$ específica para fosfatidilinositol (PI-PLC). A inativação da PI-PLC cessa a liberação de inositol 1,4,5-trifosfato e de Ca2+ intracelular, assim como a produção de diacilglicerol (DAG), o qual também ativa a proteinoquinase $\mathrm{C}^{(29)}$. Não se sabe ainda por certo como a HDL ativa a proteinoquinase $C$, porém essa ativação é importante para a inibição da ativação plaquetária ${ }^{(54)}$.

Vários autores demonstraram sítios de ligação para HDL na superfície de plaquetas, embora ainda não exista concordância entre o número e o tipo de afinidade desses receptores. O resultado pode ser devido à heterogeneidade das partículas de HDL ou ainda às incorretas interpretação e comparação entre os diversos tipos de experimentos realizados para verificar a capacidade de ligação da HDL com esses sítios de ligação. Koller et al. ${ }^{(29)}$ verificaram que a proteína de ligação para a HDL na superfície de plaquetas é idêntica à glicoproteína Ilb/Illa (GP Ilb/Illa), apesar de existirem divergências. Os receptores da família CD36 podem ser os receptores para as HDL nas plaquetas. O CD36 está intensamente presente na superfície das plaquetas e pode tanto ligar a HDL quanto outras lipoproteínas ${ }^{(54)}$. Embora exista pouca informação sobre os efeitos da HDL na função plaquetária in vivo, os resultados obtidos in vitro são bastante convincentes e importantes para o desenho e o direcionamento de novos estudos.

\section{Conclusões e perspectivas}

A dinâmica do metabolismo das lipoproteínas tem sido enfatizada nas últimas décadas como importante fator de risco cardiovascular. O conhecimento adquirido sobre a HDL e o seu papel protetor é fundamental para a otimização das estratégias terapêuticas hoje utilizadas no tratamento das alterações metabólicas passíveis de aumentar o risco de doença cardiovascular.

Inúmeras pesquisas têm demonstrado que algumas propriedades funcionais da HDL podem participar em diversos aspectos da sua atividade biológica: 1) função 
endotelial; 2) migração de monócitos; 3) proteção da oxidação da LDL; 4) ativação de fatores de coagulação (ex.: plaquetas, fator $X$, proteínas $C$ e S); 5) proliferação de células endoteliais e musculares lisas. Esse mosaico de ações pode resultar em efeitos antiinflamatórios, antioxidantes e anticoagulantes que, no conjunto, resultariam em proteção contra o desenvolvimento da aterosclerose. Por outro lado, um dos aspectos mais intrigantes que se apresenta na prática cardiológica é a constatação de eventos coronarianos em pacientes, de ambos os sexos, portadores de HDL em concentrações aceitas como protetoras. Nesse caso, quais seriam as causas da não-proteção? Haveria perda da eficiência da HDL, ou seu papel seria sobrepujado por outros fatores de risco genéticos ou adquiridos? São questões que deverão ser esclarecidas no futuro.

Continua, então, a busca por moléculas que possam atuar na elevação da concentração da HDL plasmática isoladamente baixa. Existem prováveis sítios de ação para efeitos de novos fármacos: 1) aumento na produção de apoA-l; 2) aumento da taxa de maturação da HDL; 3) diminuição do catabolismo da HDL, atuando em receptores SR-B1 hepáticos ou na CETP, sendo que neste último os dados ainda são controversos; 4) aumento da atividade de LCAT; e 5) diminuição da atividade da lipase hepática. Portanto, as investigações dirigidas às ações da HDL, na área experimental ou clínica, constituem ainda campo fértil a ser explorado.

\section{Referências}

I. ALENEZI, M. Y. et al. Is the decreased high-density lipoprotein cholesterol in the metabolic syndrome due to cellular lipid efflux defect? J Clin Endocrinol Metab, v. 89, n. 2, p. 76।-4 2004.

2. ANDERSON, T. J. Nitric oxide, atherosclerosis and the clinical relevance of endothelial dysfunction. Heart Fail Rer, v. $8, \mathrm{n}$. I, p. 7I-86, 2003.

3. ASSMANN, G; NOFER, J. R. Atheroprotective effects of highdensity lipoproteins. Annu Rev Med, v. 54, p. 321-4I, 2003.

4. BARTER, P. et al. High-density lipoproteins (HDLs) and atherosclerosis: the unanswered questions. Atherosclerosis, v. 168, n. 2, p.195-21 I, 2003.

5. BARTH, J. L.; ARGRAVES, W. S. Cubilin and megalin: partners in lipoprotein and vitamin metabolism. Trends Cardiovasc Med, v. II, n. I, p. 26-31, 2001.

6. BEHRENS,W.A. et al. Distribution of alpha-tocopherol in human plasma lipoproteins. Am J Clin Nutr, v. 35, n. 4, p. 69I-6, 1982.

7. BORGGREVE, S. E. et al. Alterations in high-density lipoprotein metabolism and reverse cholesterol transport in insulin resistance and type 2 diabetes mellitus: role of lipolytic enzymes, lecithin, cholesterol acyltransferase and lipid transfer proteins. Eur J Clin Invest, v. 33, n. 12, p. 105 I-69, 2003.

8. BRINTON, E. A. Lipid abnormalities in the metabolic syndrome. Curr Diab Rep, v. 3, n. I, p. 65-72, 2003.

9. BRUNZELL, J. D.; AYYOBI, A. F. Dyslipidemia in the metabolic syndrome and type 2 diabetes mellitus. Am J Med, v. I I5, n. I, p. 24-8, 2003

10. CANALES,A.; SANCHEZ-MUNIZ, F.J. Paraoxonase, something more than an enzyme? Med Clin (Barc), v. I2, n. 14, p. 53748, 2003.

I I. CANNON, R. O. 3rd. Role of nitric oxide in cardiovascular disease: focus on the endothelium. Clin Chem, v. 44, p. 1809-19, 1998.

12. $\mathrm{CAO}, \mathrm{H}$. et al. Paraoxonase protection of $\mathrm{LDL}$ against peroxidation is independent of its esterase activity towards paraoxon and is unaffected by the $Q \rightarrow R$ genetic polymorphism. J Lipid Res, v. 40, n. I, p. I33-9, 1999.
13. CHISOLM, G. M.; STEINBERG, D. The oxidative modification hypothesis of atherogenesis: an overview. Free Radic Biol Med, v. 28, n. 12, p. I8I5-26, 2000.

14. COCKERILL, G.W. et al. High-density lipoproteins differentially modulate cytokine-induced expression of E-selectin and cyclooxygenase-2. Arterioscler Thromb Vasc Biol, v. 19, n. 4, p. $910-7,1999$.

15. CUCHEL, M.; RADER, D.J.The role of high-density lipoproteins in thrombosis. ScientificWorldJournal, v. I2. n. 2, p. 89-95, 2002.

16. CYBULSKY, M. I. et al. A major role for VCAM-I, but not ICAM- I, in early atherosclerosis. J Clin Invest, v. 107, n. 10, p. |255-62, 200|.

17. DESAl, K. et al. Binding of apoE-rich high-density lipoprotein particles by saturable sites on human blood platelets inhibits agonist-induced platelet aggregation. J Lipid Res, v. 30, n. 6, p. 83 I-40, 1989.

18. DURRINGTON, P. N. et al. Paraoxonase and atherosclerosis. Arterioscler Thromb Vasc Biol, v. 2I, n. 4, p. 473-80, 200 I.

19. FRANCONE, O. L.; AIELLO, R. J. ABCAI: regulation, function and relationship to atherosclerosis. Curr Opin Investig Drugs, v. 3, n. 3, p. 4I5-9, 2002.

20. FREDENRICH, A.; BAYER, P. Reverse cholesterol transport, high-density lipoproteins and HDL cholesterol: recent data. Diabetes Metab, v. 29, n. 3, p. 201-05, 2003.

21. HAMMAD, S. M. et al. Cubilin, the endocytic receptor for intrinsic factor-vitamin $\mathrm{B}(\mathrm{I} 2)$ complex, mediates high-density lipoprotein holoparticle endocytosis. Proc Natl Acad Sci USA, v. 96, n. 18, p. 10158-63, 1999.

22. HAMMAD, S. M. et al. Megalin acts in concert with cubilin to mediate endocytosis of high-density lipoproteins.J Biol Chem, v. 275, n. 16, p. I2003-8, 2000.

23. HARRISON, D. G. Endothelial dysfunction in atherosclerosis. Basic Res Cardiol, v. 89, p. 87-102, 1994.

24. HIGASHIHARA, M. et al. The role of apoE in inhibitory effects of apoE-rich HDL on platelet function. FEBS Lett, v. 282, n. I, p. 82-6, I991.

25. HORIO, T. et al. Influence of low high-density lipoprotein cholesterol on left ventricular hypertrophy and diastolic 
function in essential hypertension. Am J Hypertens, v. 16, n II, p. 938-44, 2003.

26. HUANG, J. M. et al. Mechanism of high-density lipoprotein subfractions inhibiting copper-catalyzed oxidation of low-density lipoprotein. Clin Biochem, v. 31, n. 7, p. 537-43, 1998.

27. HUUSKONEN, J. et al. The impact of phospholipid transfer protein (PLTP) on HDL metabolism. Atherosclerosis, v. I 55, n. 2, p. 269-8I, 200।.

28. JIALAL, I.; DEVARAJ, S. The role of oxidized low-density lipoprotein in atherogenesis. J Nutr, v. 126, p. 1053-7, 1996.

29. KOLLER, E. et al. Purification and identification of the lipoproteinbinding proteins from human blood platelet membrane.J Biol Chem, v. 264, n. 21, p. 12412-8, 1989.

30. KONTUSH, A. et al. Small, dense HDL particles exert potent protection of atherogenic LDL against oxidative stress. ArteriosclerThromb Vasc Biol, v. 23, n. 10, p. I88 |-8, 2003.

31. KONTUSH, A. et al. Small, dense HDL particles exert potent protection of atherogenic LDL against oxidative stress. Arterioscler Thromb Vasc Biol, v. 23, n. 10, p. I 881-8, 2003.

32. KOPPAKA, V. Structural studies of discoidal lipoprotein A-I. Cell Mol Life Sci, v. 58, n. 7, p. 885-93, 2001.

33. KUME, N. et al. Lysophosphatidyl-choline, a component of atherogenic lipoproteins, induces mononuclear leukocyte adhesion molecules in cultured human and rabbit arterial endothelial cells. J Clin Invest, v. 90, n. 3, p. I I 38-44, 1992.

34. KUVIN, J.T. et al. A novel mechanism for the beneficial vascular effects of high-density lipoprotein cholesterol: enhanced vasorelaxation and increased endothelial nitric oxide synthase expression. Am Heart J, v. I 44, n. I, p. I65-72, 2002.

35. LAGGNER, P. et al. Separation of subclasses of human serum high-density lipoproteins by zonal ultracentrifugation. Prep Biochem, v. 7, n. I, p. 33-43, 1977.

36. LESNIK, P. et al. Anti-coagulant activity of tissue factor pathway inhibitor in human plasma is preferentially associated with dense subspecies of LDL and HDL and with Lp(a). Arteriosd Thromb, v. 13, n. 7, p. 1066-75, 1993.

37. LULIANO, L. The oxidant stress hypothesis of atherogenesis Lipids, v. 36, p. 4l-4, 200 I.

38. LUNDASEN, T. et al. Leptin induces the hepatic high-density lipoprotein receptor scavenger receptor B type I (SR-BI) but not cholesterol 7alpha-hydroxylase (Cyp7al) in leptin-deficient (ob/ob) mice.J Biol Chem, v. 278, n. 44, p. 43224-8, 2003.

39. LUND-KATZ, S. et al. High density lipoprotein structure. Front Biosci, v. 8, p. 1044-54, 2003.

40. LUPATTELLI, G. et al. Mechanisms of high-density lipoprotein cholesterol effects on the endothelial function in hyperlipemia. Metabolism, v. 52, n. 9, p. | | 9|-5, 2003.

4I. MACKNESS, B. et al. Polymorphisms of paraoxonase genes and low-density lipoprotein lipid peroxidation. Lancet, v. 353, n. 915I, p. 468-9, 1999.

42. MACKNESS, M. I. et al. How high-density lipoprotein protects against the effects of lipid peroxidation. Curr Opin Lipidol, v. I I, n. 4, p. 383-8, 2000.

43. MACKNESS, M. I. et al. Low serum paraoxonase: a risk factor for atherosclerotic disease? Chem Biol Interact, v. I 19-20, p. 389-97, 1999.

44. MACKNESS, M. I. et al. Paraoxonase prevents accumulation of lipoperoxides in low-density lipoprotein. FEBS Lett, v. 286, n. |-2, p. |52-4, 199|.
45. MACKNESS, M. I. et al.The role of high-density lipoprotein and lipid-soluble antioxidant vitamins in inhibiting low-density lipoprotein oxidation. Biochem J, v. 294, p. 829-34, 1993.

46. MACKNESS, M. I.; DURRINGTON, P. N. HDL, its enzymes and its potential to influence lipid peroxidation. Atherosclerosis, v. I I 5, n. 2, p. 243-53, 1995.

47. MARSH, J. B. Lipoprotein metabolism in obesity and diabetes: insights from stable isotope kinetic studies in humans. Nutr Rev, v. 6I, n. II p. 363-75, 2003.

48. MINEO, C. et al. High-density lipoprotein-induced endothelial nitric-oxide synthase activation is mediated by Akt and MAP kinases. J Biol Chem, v. 278, n. I I, p. 9|42-9, 2003.

49. MINEO, C.; SHAUL, P.W. HDL stimulation of endothelial nitric oxide synthase: a novel mechanism of HDL action. Trends Cardiovasc Med, v. I3, n. 6, p. 226-31, 2003.

50. MOESTRUP, S. K.; KOZYRAKI, R. Cubilin, a high-density lipoprotein receptor. Curr Opin Lipidol, v. I I, n. 2, p. I33-40, 2000.

5 I. MOYER, M. P. et al. Plasma lipoproteins support prothrombinase and other procoagulant enzymatic complexes. Arterioscler Thromb Vasc Biol, v. I 8, n. 3, p. 458-65, 1998

52. $\mathrm{NAZIH}, \mathrm{H}$. et al. Protein kinase $\mathrm{C}$-dependent desensitization of HDL3 activated phospholipase $C$ in human platelets. ArteriosclerThromb, v. 14, n. 8, p. 1321-6, 1994

53. NEUFELD, M. et al. High-density lipoproteins inhibit fibrinogen binding on adenosine diphosphate-activated monocytes. Blood Coagul Fibrinolysis, v. I I, n. 6, p. 505-9, 2000.

54. NOFER, J. R. et al. HDL and arteriosclerosis: beyond reverse cholesterol transport. Atherosclerosis, v. I6 I, n. I, p. I- 16, 2002

55. NOFER, J. R. et al. HDL3-mediated inhibition of thrombininduced platelet aggregation and fibrinogen binding occurs via decreased production of phosphoinositide-derived second messengers 1,2-diacylglycerol and inositol 1,4,5-trisphosphate. Arterioscler Thromb Vasc Biol, v. I8, p. 86I-9, 1998.

56. O'CONNELL, B. J.; GENEST, J. Jr. High density lipoproteins and endothelial function. Circulation, v. 104, n. 16, p. 1978-83, 2001 .

57. ORAM,J.F.ATP-binding cassette transporter $\mathrm{AI}$ and cholesterol trafficking. Curr Opin Lipidol, v. I3, n. 4, p. 373-81, 2002.

58. OSEROFF, A. et al. Plasminogen activator and plasminogen activator inhibitor activities in men with coronary artery disease. J Lab Clin Med, v. I I 3, n. I, p. 88-93, 1989.

59. OTVOS, J. D. Measurement of lipoprotein subclass profile by nuclear magnetic resonance spectroscopy. In: WARNICK, R.; DOMINICZAK, M. Handbook of lipoprotein testing. Washington: AACC Press. 2000. p. 609-23.

60. OTVOS, J. D. Measurement of lipoprotein subclass profile by nuclear magnetic resonance spectroscopy. Clin Lab, v. 48, n. 3-4, p. I7I-80, 2002

6I. PERUGINI, C. et al. Distribution of lipid-soluble antioxidants in lipoproteins from healthy subjects. I. Correlation with plasma antioxidant levels and composition of lipoproteins. Pharmacol Res, v. 4I, n. I, p. 55-65, 2000.

62. PERUGINI, C. et al. Distribution of lipid-soluble antioxidants in lipoproteins from healthy subjects. II. Effects of in vivo supplementation with alpha-tocopherol. Pharmacol Res, v. 4I, n. I, p. 67-74, 2000.

63. PRIMO-PARMO, S. L. et al. The human serum paraoxonase/ arylesterase gene $(\mathrm{PONI})$ is one member of a multigene family. Genomics, v. 33, n. 3, p. 498-507, 1996. 
64. PRINTSEVA, O.Y. et al.Various cell types in human atherosclerotic lesions express ICAM-I. Further immunocytochemical and immunochemical studies employing monoclonal antibody IOF3. Am J Pathol, v. I40, n. 4, p. 889-96, 1992.

65. RAINWATER, D. L. Electrophoretic separation of LDL and HDL subclasses. Methods Mol Biol, v. I I0, p. I37-5I, 1998.

66. RAINWATER, D. L. et al. Characterization of a composite gradient gel for the electrophoretic separation of lipoproteins.J Lipid Res, v. 38, n. 6, p. 1261-6, 1997

67. RAMET, M. E. et al. High-density lipoprotein increases the abundance of eNOS protein in human vascular endothelial cells by increasing its half-life. J Am Coll Cardiol, v. 4I, n. 12, p. 2288-97, 2003.

68. RASHID, S. et al. Mechanisms of HDL lowering in insulin resistant, hypertriglyceridemic states: the combined effect of HDL triglyceride enrichment and elevated hepatic lipase activity. Clin Biochem, v. 36, n. 6 p. 421-9, 2003.

69. RIDDELL, D. R. et al.Apolipoprotein E inhibits platelet aggregation through the L-arginine: nitric oxide pathway. Implications for vascular disease.J Biol Chem, v. 272, n. I, p. 89-95, 1997.

70. RIDDELL, D. R. et al. Identification and characterization of LRP8 (apoER2) in human blood platelets. J Lipid Res, v. 40, n. 10, p. 1925-30, 1999.

7I. RUIZ, J. et al. Gln-Arg 192 polymorphism of paraoxonase and coronary disease in type 2 diabetes. Lancet, v. 346, p. 869 72, 1995.

72. RUOTOLO, G.; HOWARD, B.V. Dyslipidemia of the metabolic syndrome. Curr Cardiol Rep, v. 4, n. 6, p. 494-500, 2002.

73. RYE, K. A.; BARTER, P. J. Formation and metabolism of prebetamigrating, lipid-poor apolipoprotein A-I. Arterioscler Thromb Vasc Bio, v. 24, n. 3, p. 421-8, 2004

74. SAKUMA, N. et al. HDL3 exerts a more powerful antiperoxidative and protective effect against peroxidative modification of LDL than HDL2 does. J Nutr Sci Vitaminol (Tokyo), v. 48, n. 4, p. 278-82, 2002.

75. SCHILLACl, G. et al. High-density lipoprotein cholesterol and left ventricular hypertrophy in essential hypertension. J Hypertens, v. 19, n. 12, p. 2265-70, 2001.

76. SCHMITZ, G.; BUECHLER, C. ABCAI: regulation, trafficking and association with heteromeric proteins. Ann Med, v. 34 n. 5, p. 334-47, 2002.

77. SEGREST, J. P. et al. Structure and function of apolipoprotein A-I and high-density lipoprotein. Curr Opin Lipidol, v. I I, n. 2, p. I05- I5, 2000.

78. SERRATO, M.; MARIAN, A. J. A variant of human paraoxonase/ arylesterase (HUMPONA) gene is a risk factor for coronary artery disease.J Clin Invest, v. 96, n. 6, p. 3005-8, 1995.

79. SINGH, K. et al. High density lipoprotein subclasses inhibit lowdensity lipoprotein oxidation. Indian J Biochem Biophys, v. 34, n. 3, p. 3|3-8, 1997.

80. SMITH, J. D. et al. ABCAI mediates concurrent cholesterol and phospholipid efflux to apolipoprotein A-I. J Lipid Res, v. 45, n. 4, p. 635-44, 2004.

8I. SPIEKER, L. E. et al. High-density lipoprotein restores endothelial function in hypercholesterolemic men. Circulation, v. I05, n. 12, p. I 399-402, 2002

82. SRIVASTAVA, N. ATP-binding cassette transporter Al: key roles in cellular lipid transport and atherosclerosis. Mol Cell Biochem, v. 237, n. I-2, p. I55-64, 2002.

83. STEINBERG, D. Oxidative modification of $L D L$ in the pathogenesis of atherosclerosis. Am J Geriatr Cardiol, v. 2, n. 5, p. 38-4I, 1993.

84. TAILLEUX, A. et al. Apolipoprotein A-II, HDL metabolism and atherosclerosis. Atherosclerosis, v. I 64, n. I, p. I - 13, 2002.

85. TALL, A. R. et al. Changes in the distribution and composition of plasma high-density lipoproteins after ingestion of fat. J Biol Chem, v. 257, n. I, p. 198-207, 1982.

86.TRACY, R. P.Thrombin, inflammation, and cardiovascular disease: an epidemiologic perspective. Chest, v. 124, p. 49-57, 2003.

87. TRIGATTI, B. L. et al. Influence of the HDL receptor SR-BI on lipoprotein metabolism and atherosclerosis. Arterioscler Thromb Vasc Biol, v. 23, n. 10, p. 1732-8, 2003.

88. VON ECKARDSTEIN, A. et al. High-density lipoprotein and atherosclerosis: role of cholesterol efflux and reverse cholesterol transport. Arterioscler Thromb Vasc Biol, v. 2 I, n. I, p. 13-27, 2001.

89.WANG, M.; BRIGGS, M. R. HDL: the metabolism, function, and therapeutic importance. Chem Rev, v. 104, n. I, p. I19-37, 2004.

90.WANG, N. et al.ATP-binding cassette transporter AI (ABCAI) functions as a cholesterol efflux regulatory protein. J Biol Chem, v. 276, n. 26, p. 23742-7, 2001.

9।. WANG, N.; TALL, A. R. Regulation and mechanisms of ATP-binding cassette transporter AI-mediated cellular cholesterol efflux. Arterioscler Thromb Vasc Biol, v. 23, n. 7, p. I 178-84, 2003.

92.WARNICK, G. R. et al. Evolution of methods for measurement of HDL-Cholesterol: from ultracentrifugation to homogeneous assays. Clin Chem, v. 47, n. 9, p. 1579-96, 2001.

93.WATSON,A. D. et al. Protective effect of high-density lipoprotein associated paraoxonase: inhibition of the biological activity of minimally oxidised low-density lipoprotein. J Clin Invest, v. 96, n. 6, p. 2882-89।, 1995.

94.WATSON, K. E. et al. Lipid abnormalities in insulin resistant states. Rev Cardiovasc Med, v. 4, n. 4, p. 228-36, 2003.

95. WESTHUYZEN, J.The oxidation hypothesis of atherosclerosis: an update. Ann Clin Lab Sci, v. 27, n. I, p. I - 10, 1997.

96. YOUNG, C. E. et al. High-density lipoprotein cholesterol and coronary heart disease. Cardiol Rev, v. 12, n. 2, p. 107-19, 2004.

97. YUHANNA, I. S. et al. High-density lipoprotein binding to scavenger receptor-BI activates endothelial nitric oxide synthase. Nat Med, v. 7, n. 7, p. 853-7, 200 I.

Endereço para correspondência 